\title{
Electron Paramagnetic Resonance Spectroscopic Investigation of the Dynamics of Spin Probe in Room Temperature Ionic Liquid
}

Arijit Sengupta* and Kadam RM

Bhabha Atomic Research Centre, Trombay, Mumbai, Maharashtra, India

\begin{abstract}
Rotational motion of the spin probe 4-hydroxyl-2,2,6,6- tetramethyl-1-piperidinyloxy (TEMPOL) in methyl imidazoium based ionic liquid was studied by Electron Paramagnetic Resonance spectroscopy (EPR). The influence of rotational tumbling of the paramagnetic spin probe on the width and shape of EPR transition was studied to calculate rotational correlation time, equilibrium radius, and rotational diffusion coefficient of spin probe in different ionic liquid media; $\mathrm{C}_{\mathrm{n} \text { mimPF}} / \mathrm{NTf}_{2}(\mathrm{n}=4,6,8)$. The glass transition temperatures for $\mathrm{C}_{\mathrm{m}} \operatorname{mimNTf}_{2}(\mathrm{n}=4,6)$ were calculated from the inflection point of $T_{c}$ vs $T$ plots whereas activation energy of the rotation were calculated from the Arrhenius plots of InD vs $1 / \mathrm{T}(\mathrm{K})$.
\end{abstract}

Keywords: Dynamics; Spin probe; Correlation time; EPR spectroscopy; TEMPOL; Arrhenius equation

\section{Introduction}

On the growing awareness of the potential hazards associated with the use of conventional molecular solvent, there is a need of alternative environmentally benign diluents. Room temperature ionic liquids have been considered as alternative 'green solvents' to the conventional molecular diluents in several fields like separation science, electrochemistry, synthesis, catalysis etc due to their attractive properties of the former like low vapour pressure, wide liquid range, solubility of wide range of organic, inorganic and polymeric materials, relatively large potential window, etc. In nuclear industry, several attempts were also made to use trill along with extractants as organic phase for metal ion separation from waste stream [1-8] or use of functionalized ionic liquid [9-13] (for the same. Because of large potential window, ionic liquid is also used for electrochemical studies [14-17] Room temperature ionic liquid showed very promising extraction properties of actinides compared to molecular diluents. However, one of the major drawbacks of using ionic liquid is slower extraction kinetics because of its high viscosity coefficient. The extraction and separation process is highly influenced by the diffusion properties of metal ions; ligands etc. through the ionic liquid medium and solvating ability of the new class of the RTIL. Therefore, Electron Paramagnetic Resonance (EPR) studies were conducted to understand the molecular dynamics of spin probe in RTIL media. An attempt was made to correlate the changes in cationic and anionic moieties on the molecular dynamics of spin probe and physicochemical properties of the ionic liquid media which have significant role in separation science as well as electrochemistry.

Although a few studies have been completed on the dynamics of ionic liquids [18-21] only a few studies were found in the literature on electron paramagnetic spin probe in RTIL. In the present study, stable free radical 4-hydroxy-2, 2, 6, 6-tetramethylammoniumpiperidine-1oxyl (TEMPOL) was selected as the diffusional probe for this study as it possess simple and well characterized EPR spectrum and consequently information on both translational and rotational diffusion of this molecule may be obtained by investigating the line shape of the EPR signals as a function of temperature which is needed for understanding the transport properties and complexation ability of this new class of molecular solvents. The correlation time $\left(\tau_{c}\right)$, diffusion coefficient (D) of the spin probe and its equilibrium radius $(r)$ in different RTIL were investigated at room temperature. From the temperature variation EPR spectra, the 'glass transition temperature $\left(\mathrm{T}_{\mathrm{g}}\right.$ )' and the activation energy $\left(E_{a}\right)$ for diffusion of the spin probe were evaluated for $\mathrm{C}_{n} \operatorname{mimNTf}_{2}(\mathrm{n}=4$ and 6).

\section{Experimental}

EPR studies were carried out using a Bruker-EMX (EMM1843) spectrometer at X-band frequency operated at $9.5 \mathrm{GHz}$ with $100 \mathrm{kHz}$ field modulation. The ' $\mathrm{g}$ ' values were evaluated relative to a small single crystal of BDPA (a,v bisdiphenylene -B-phenyallyl) with $\mathrm{g}=2.0028$ and a line width of 0.63 Gauss. Temperature variation studies were done on a Bruker variable temperature accessory Eurotherm BVT2000. $\mathrm{C}_{\mathrm{n}} \operatorname{mimNTf}_{2} / \mathrm{PF}_{6}$ (where $\left.\mathrm{n}=4,6,8\right)>99 \%$ purity was obtained from Ioli Tech, Germany and was used after ensuring the absence of moisture $(<0.1 \%)$. The stable nitroxide radicals 4 -hydroxy-2,2,6,6tetramethylpiperidine-1-oxyl (Fulka, $\geq 97 \%$ ) was employed as spin probes. Samples were prepared gravimetrically; i.e., values and molar concentrations were calculated from literature densities [22]. The concentration of spin label was taken as $5 \times 10^{-3} \mathrm{M}$. The sample solutions were transferred into borosilicate glass capillary tubes with a bore diameter of $0.8 \mathrm{~mm}$ under an argon atmosphere, subjected to three freeze - pump - thaw cycles, and sealed off under vacuum.

\section{Results and Discussion}

The main information provided by the EPR of nitroxide probes are the determination of the local viscosity if the radical is rigid or the dynamics of segmental motions if it is attached to a flexible molecule. The tumbling motion, the translational diffusion on a curved surface as well as the exchange of the probe among rigid and fluid sites result in a reduction of the anisotropic components of the magnetic tensors to finite values if the medium is organized (liquid crystal) or isotropic and viscous. In an isotropic fluid these components are averaged out. Accordingly, as the molecular tumbling is isotropic or not, the re orientational motion is characterized by one or several correlation times. In the following, the geometrical mean of the re-orientation correlation time about the principal axes of the rotational diffusion tensor will be defined as the effective correlation time. The nitroxide radicals are of widespread use as spin labels or spin probes in the

*Corresponding author: Arijit Sengupta, Bhabha Atomic Research Centre, Trombay, Mumbai-400 085, Maharashtra, India, Tel: 02225505050; E-mail: arijita@barc.gov.in

Received September 02, 2016; Accepted September 18, 2016; Published September 22, 2016

Citation: Sengupta A, Kadam RM (2016) Electron Paramagnetic Resonance Spectroscopic Investigation of the Dynamics of Spin Probe in Room Temperature Ionic Liquid. Mod Chem Appl 4: 189. doi: 10.4172/2329-6798.1000189

Copyright: $\odot 2016$ Sengupta A, et al. This is an open-access article distributed under the terms of the Creative Commons Attribution License, which permits unrestricted use, distribution, and reproduction in any medium, provided the original author and source are credited. 
applications of EPR to physical-chemistry and biophysics on account of their thermal stability and of the significant anisotropy of the A and $\mathrm{g}$ tensors Figure 1.

\section{Effect of modification in cationic and anionic moieties of ionic liquids on the mobility of the spin probe}

One of the interesting properties of the room temperature ionic liquids is their tunability i.e., small structural modifications in the cationic and anionic moieties in the ionic liquid can lead to drastic changes in the physical chemical properties of ionic liquids. It was observed that for imidazolium based ionic liquids, with increasing chain length of alkyl substituents the extraction kinetics become slower [23] and the potential window extends to the more negative potential value $[15,16]$. It was also reported that with increasing chain length of the alkyl substituents, the extraction mechanism changes from ion exchange to salvation [24]. Similarly, effect of anion on either separation science or in electro-chemistry was also studied $[15,16]$. Though there are a few literature reports are available which may help one to understand the motions of spin probes in ionic liquid [19,20,22], a systematic study was carried out to understand the structural modifications of ionic liquid on the motion of spin probe into the ionic liquid. Figure 2 shows the room temperature (300K) EPR spectra of the above mentioned spin probe in different ionic liquids. The isotropic nature of the spectra revealed that the spin probes are in complete motion in all the ionic liquids at $300 \mathrm{~K}$. The correlation time for motion of spin probe, $\tau_{\text {, }}$, is typically the Stockes-Debye-Einstein rotational correlation time is given by the equation $[22,25,26]$,

$$
\tau_{\mathrm{R}}=1 / 6 \mathrm{D}_{\mathrm{R}}=4 \pi \mathrm{r}^{3} \eta / 3 \mathrm{k}_{\mathrm{B}} \mathrm{T}
$$

Where $D_{R}$ and $\tau_{R}$ are termed as the rotational diffusion coefficient and the rotational correlation time, $r$ is the hydrodynamic radius, $\eta$ is the micro viscosity, $\mathrm{T}$ is absolute temperature and $\mathrm{k}_{\mathrm{B}}$ is the Boltzmann constant. $\mathrm{H}$ is higher in the 'water phase of the membrane than in the bulk aqueous solution. It may be noted that there is an inverse relation exist between rotational diffusion coefficient and viscosity of solvent/ medium. For sufficiently viscous solvents, EPR spectroscopy is used to measure the rotational coefficient of spin probe by analyzing the spectral line shapes, a technique which exploits the fact that restricted molecular tumbling of the rotational probe leads to an incomplete averaging of the EPR signal on the experimental time scale, which in term leads to an asymmetry in the resulting spectrum. For nitroxide with $S=1 / 2$ in fluid solution the EPR line shapes are governed by two

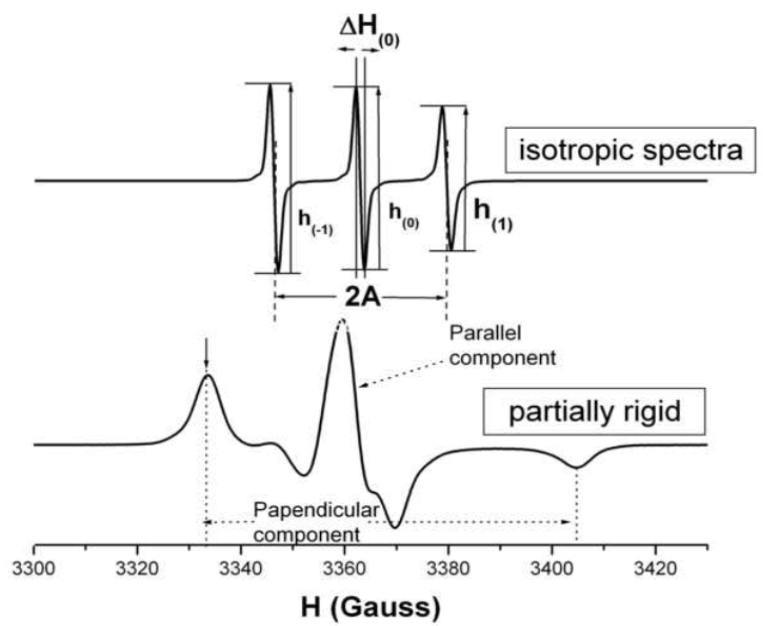

Figure 1: $\mathrm{X}$ band spectrum of the TEMPOL radical computed with $\mathrm{g}_{\mathrm{xx}}=2.0096$, $g_{y y}=2.0058, g_{z z}=2.0022, a_{x x}=7, a_{y y}=6, a_{z z}=36 \mathrm{G}$. main mechanisms: spin rotation and modulation of the magnetic anisotropies. If the reorientation time of the relaxing species $\left(\mathrm{T}_{2}\right)$ is short with respect to the various anisotropies, $\mathrm{T}_{2}^{-1}$ is given by equation

$$
\mathrm{T}_{2(\mathrm{mI})}^{-1}=\alpha+\alpha^{\prime}+\beta \mathrm{m}_{\mathrm{I}}+\gamma \mathrm{m}_{\mathrm{I}}^{2}
$$

More detailed information about the radical mobility were obtained from the analysis of the spectral line shapes and line widths in terms of a correlation times $(\tau)$ and hence on the experimental temperature $[25,26]$.

$$
\begin{aligned}
& \tau_{\mathrm{c}}=\left(6.5 \times 10^{-10}\right) \Delta \mathrm{H}_{(0)}\left\{\left[\mathrm{h}_{(0)} / \mathrm{h}_{(-1)}\right]^{1 / 2}-\left[\mathrm{h}_{(0)} / \mathrm{h}_{(1)}\right]^{1 / 2}\right\} \\
& \tau_{\mathrm{B}}=\left(6.5 \times 10^{-10}\right) \Delta \mathrm{H}_{(0)}\left\{\left[\mathrm{h}_{(0)} / \mathrm{h}_{(1)}\right]^{1 / 2}+\left[\mathrm{h}_{(0)} / \mathrm{h}_{(-1)}\right]^{1 / 2}-2\right\}
\end{aligned}
$$

Where $6.5 \times 10^{-10}$ is an averaged parameter that takes in to account A and $g$ anisotropies, $\Delta \mathrm{H}_{(0)}$ is the actual line width inside the central manifold and $h(1), h(0)$ and $h(-1)$ corresponds to the intensities of low field, center and high field lines corresponding to $m_{I}=+1,0$ and -1 , respectively. The width $\Delta \mathrm{H}_{(\mathrm{m})}(\mathrm{m}=1,0,-1)$ of the individual hyperfine line is given by

$$
\Delta \mathrm{H}_{(\mathrm{m})}=\mathrm{A}+\mathrm{Bm}_{\mathrm{I}}+\mathrm{Cm}_{\mathrm{I}}^{2}
$$

In case of anisotropic motion $\tau_{c} \neq \tau_{B}$, with the motion rate along $\mathrm{z}$ axis faster than along the $\mathrm{x}$ and $\mathrm{y}$ axis. When condition $\tau_{\mathrm{c}} \approx \tau_{\mathrm{B}}$ is fulfilled the motion can be treated as isotropic $\left(10^{-11}<\tau<5 \times 10^{-9}\right)$. Table 1 summerizes the correlation time, rotational diffusion coefficient, and equilibrium radius of spin probes in different ionic liquids. It was observed that with increase the chain length of alkyl substituent, the viscosity coefficients of the RTIL medium increases [23]. Consequently, the motion of spin probe is restricted and it requires longer time to complete a single rotation. Similar trend is observed if the anionic counterpart i.e., $\mathrm{NTf}_{2}$ is changed to $\mathrm{PF}_{6}^{-}$due to viscosity effect. The rotational coefficient for TEMPO in the six ionic liquids at room temperature was found in the range $1.27 \times 10^{-8} \mathrm{~s}^{-1}-7.82 \times 10^{-8} \mathrm{~s}^{-1}$. The rotational correlation time of imidazolim based ionic liquids with $\mathrm{BF}_{4}$ as anion was reported to be in the range of $130-770 \mathrm{ps}$ [25] while that of $\mathrm{C}_{8} \operatorname{mimPF}_{6}$ calculated in present case was found to be similar with that reported in the literature [25]. The equilibrium radius of spin probe was found to be $\sim 1.3 \mathrm{~A}$. A slight variation can be due to the difference in the interaction of the spin probes with the ionic liquid.

Temperature dependence EPR spectra of spin probe, determination of glass transition temperature, activation energy

The ESR spectra of the RTIL with TEMPO were recorded over a wide temperature range of 100-300 K and they are shown in Figure 3. As the viscosity of the samples increases with decreasing the temperature, the rotation of the TEMPO molecule become increasingly restricted and the anisotropy in the hyperfine coupling constants and $g$ value are less effectively averaged out. As evident from the Figure 3 , the EPR spectra were increasing asymmetric at about $260 \mathrm{~K}$. The motion of the spin probe in the temperature range $350-300 \mathrm{~K}$, corresponds to fast thumbing region $10^{-10}<\tau_{\mathrm{R}}<5 \times 10^{-9} \mathrm{sec}$. In the intermediate temperature range $300-220 \mathrm{~K}$, EPR spectrum became relatively broad and progressively asymmetric on lowering the temperature. This region corresponds to slow thumbing region $5 \times 10^{-9}<\tau_{\mathrm{R}}<50 \times 10^{-9} \mathrm{sec}$. And on further cooling these room temperature ionic liquids at $100 \mathrm{~K}$, all types of molecular motions (translational and rotational motion) of TEMPOL are completely frozen yielding an axially symmetric spectrum corresponding to time scale of rotation $\sim 10^{-6} \mathrm{sec}$ which could be assumed to be in rigid limits. The rotational correlation time can be estimated by analyzing the EPR line widths and the relative intensities (line shapes). 
Citation: Sengupta A, Kadam RM (2016) Electron Paramagnetic Resonance Spectroscopic Investigation of the Dynamics of Spin Probe in Room Temperature lonic Liquid. Mod Chem Appl 4: 189. doi: 10.4172/2329-6798.1000189

Page 3 of 5

\begin{tabular}{|c|c|c|c|c|}
\hline Ionic liquid & $\mathbf{T}(\mathrm{ps})$ & $D\left(s^{-1}\right)$ & $\eta(\mathrm{mPa} . \mathrm{S})$ & $r_{e}(A)$ \\
\hline $\mathrm{C}_{4} \mathrm{mim}^{+} \mathrm{NTf}_{2}^{-}$ & 213.00 & $7.82 \mathrm{E}+08$ & 46.41 & 1.66 \\
\hline $\mathrm{C}_{4} \mathrm{mim}^{+} \mathrm{PF}_{6}^{-}$ & 316.50 & $5.27 \mathrm{E}+08$ & 250.16 & 1.08 \\
\hline $\mathrm{C}_{6} \mathrm{mim}^{+} \mathrm{NTf}_{2}^{-}$ & 229.50 & $7.26 \mathrm{E}+08$ & 52.02 & 1.63 \\
\hline $\mathrm{C}_{6} \mathrm{mim}^{+} \mathrm{PF}_{6-}$ & 421.50 & $3.95 \mathrm{E}+08$ & 308.56 & 1.11 \\
\hline $\mathrm{C}_{8} \mathrm{mim}^{+} \mathrm{NTf}_{2}^{-}$ & 240.00 & $6.94 \mathrm{E}+08$ & 72.64 & 1.48 \\
\hline $\mathrm{C}_{8} \mathrm{mim}^{+} \mathrm{PF}_{6}^{-}$ & 1311.00 & $1.27 \mathrm{E}+08$ & 694.25 & 1.23 \\
\hline
\end{tabular}

Table 1: Determination of correlation time ( $T$ ), diffusion coefficient $(D)$ and equilibrium radius (re) of spin probes in different ionic liquid.

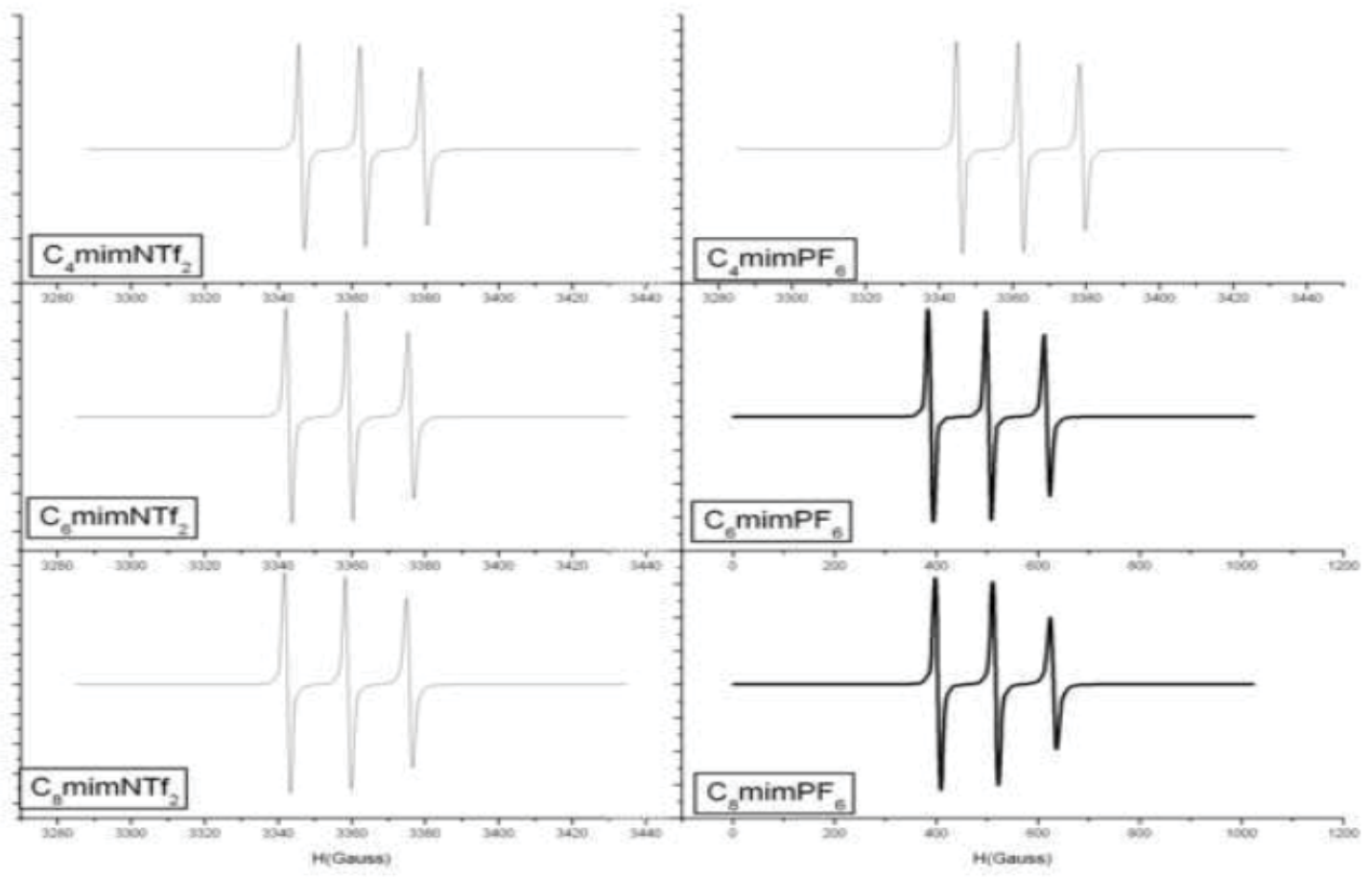

Figure 2: Isotropic EPR spectra of spin probe in different ionic liquids at 300K.
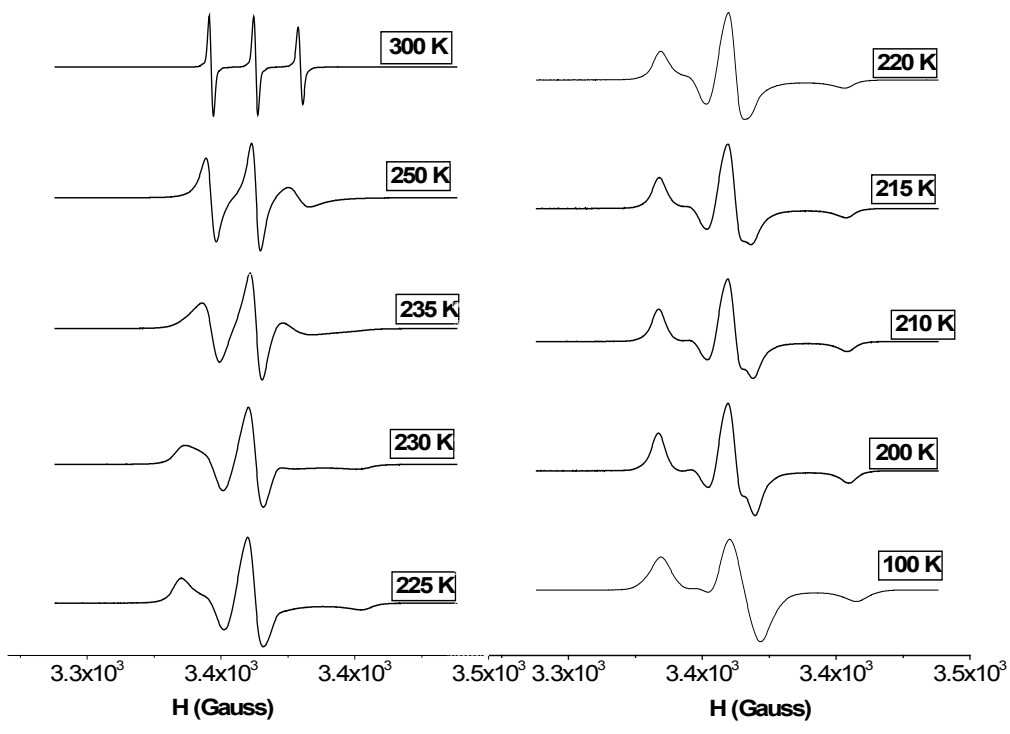

Figure 3: Temperature dependent EPR spectra of spin probe in $\mathrm{C}_{4} \operatorname{mimNTf}_{2}$. 
Figure 3 showed the temperature dependent EPR spectra of $\mathrm{C}_{4} \operatorname{mimNTf}_{2}$ in the range $100 \mathrm{~K}-300 \mathrm{~K}$. The EPR spectra at $100 \mathrm{~K}$ and $300 \mathrm{~K}$ are entirely different, as $300 \mathrm{~K}$ spectra is isotropic spectra obtained due to fast motion of spin probe into the medium, while 100 $\mathrm{K}$ spectra is completely resolved anisotropic spectra representing the rigidity of the spin probe. As the temperature of the system decreased the motion of the spin probe is restricted and anisotropy in the EPR spectra arises. Glass transition temperature is one of the important parameters for a medium. Monitoring the motion of spin probe by EPR spectra in ionic liquid, attempt was made to determine the glass transition temperature of $\mathrm{C}_{n} \operatorname{mimNTf}_{2}(\mathrm{n}=4,6)$. Figure 4 showed a plot of correlation time at different temperature for these ionic liquids mentioned above. It was observed that on lowering of temperature, the correlation time increases gradually as the motion of the spin probe is getting restricted. a sharp increase in $\tau_{c}$ values is noticed at a particular temperature interval. The temperature at which the inflection point observed is termed as glass transition temperature. For $\mathrm{C}_{4}$ mimNTf $_{2}$ the glass transition temperature was determined as $215 \mathrm{~K}$ whereas for
$\mathrm{C}_{6} \operatorname{mimNTf}_{2}$ it is $225 \mathrm{~K}$ (Table 2). Form $\tau_{c}$ values the diffusion coefficient for the rotation can be calculated at various temperature as shown by eqn (2). The diffusion coefficient (D) and the activation energy $\left(\mathrm{E}_{\text {act }}\right)$ can be related by the well-known Arhenious equation as follows $[25,26]$

$$
\mathrm{D}=\mathrm{A} \exp \left(\mathrm{E}_{\mathrm{act}} / \mathrm{RT}\right)
$$

A plot of $\ln \mathrm{D}$ as a function of $\mathrm{T}$ should give a straight line. From the slope the activation energy values are determined. More viscous the medium is, less will be the $\mathrm{D}$ value and more will be the $\mathrm{E}_{\text {act }}$ value as signature in Table 2. The Arrhenius plot is shown in Figure 5.

\section{Conclusions}

An attempt was made to correlate the structural modifications of imidazolium based ionic liquids on the mobility of spin probe which is important to understand the mechanism of any reaction in ionic liquid media. It was observed that on increasing the length of alkyl substituent in methyl imidazolium moiety, the mobility of spin probe gets freezed, correlation time becomes more. This fact can be attributed to the

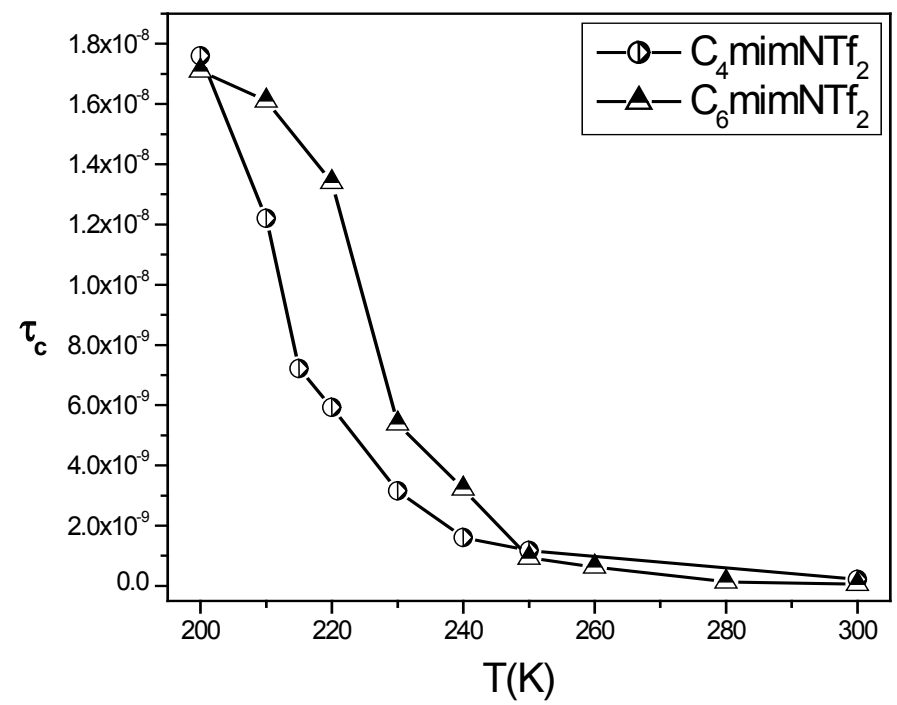

Figure 4: Plots of correlation time as a function of temperature.

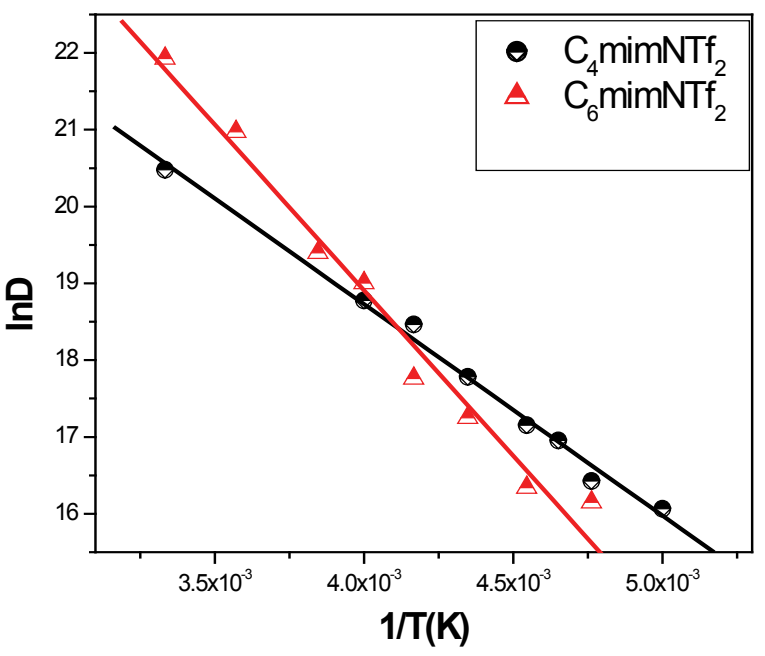

Figure 5: Arrhenius plots for $C_{n} \operatorname{mimNTf}_{2}(n=4,6)$. 
Citation: Sengupta A, Kadam RM (2016) Electron Paramagnetic Resonance Spectroscopic Investigation of the Dynamics of Spin Probe in Room Temperature Ionic Liquid. Mod Chem Appl 4: 189. doi: 10.4172/2329-6798.1000189

\begin{tabular}{|c|c|c|}
\hline Medium & $\mathbf{T}_{\mathbf{g}}(\mathbf{K})$ & $\mathbf{E}_{\text {act }}\left(\mathbf{k J m o l}^{-1}\right)$ \\
\hline $\mathrm{C}_{4}$ mimNTf $_{2}$ & $215 \mathrm{~K}$ & $22.89 \pm 0.78$ \\
\hline $\mathrm{C}_{6} \mathrm{mimNTf}_{2}$ & $225 \mathrm{~K}$ & $31.60 \pm 2.86$ \\
\hline
\end{tabular}

Table 2: Determination of glass transition temperature $\left(T_{g}\right)$ and activation energy $\left(E_{\text {act }}\right)$ for $\mathrm{C}_{n} \operatorname{mimNTf}_{2}(n=4,6)$.

viscosity effect. Similar observation was also seen in case of changing the anionic parts of the ionic liquids from $\mathrm{NTf}_{2}^{-}$to $\mathrm{PF}_{6}^{-}$(which leads to the increase in viscosity coefficient of the medium). The rotational coefficient, solvodynamic radius of spin probe was calculated for $\mathrm{C}_{\mathrm{n}} \operatorname{mimPF}_{6} / \mathrm{NTf}_{2}(\mathrm{n}=4,6,8)$. From Arrhenius plot the activation energy for rotation of the spin probe and glass transition temperatures were also evaluated.

\section{References}

1. Binnemans $\mathrm{K}$ (2007) Lanthanides and Actinides in lonic Liquids. Chem Rev 107: 2592-2614

2. Billard I (2013) In: Handbook on the Physics and Chemistry of Rare Earths. Bünzli JCG, Pecharsky V (éds). Elsevier Science Publication, Amsterdam 43: 26.

3. Rao PVR, Venkatesan KA, Alok R, Srinivasan TG, Nagarajan K, et al. (2012) Potential applications of room temperature ionic liquids for fission products and actinide separation. Sep Sci Technol 47: 204-222.

4. Kolarik Z (2013) Solv Extr Ion Exch 31: 24-60.

5. Sun X, Luo H, Dai S (2012) lonic Liquids-Based Extraction: A Promising Strategy for the Advanced Nuclear Fuel Cycle. Chem Rev 112: 2100-2128.

6. Mohapatra PK, Sengupta A, Iqbal M, Huskens J, VerboomW, et al. (2013) Diglycolamide-functionalized calix[4]arenes showing unusual complexation of actinide ions in room temperature ionic liquids: role of ligand structure, radiolytic stability, emission spectroscopy, and thermodynamic studies. Inorg Chem 52: 2533-2541.

7. Sengupta A, Mohapatra PK, Iqbal M, Huskensb J, Verboom W, et al. (2012) A highly efficient solvent system containing functionalized diglycolamides and an ionic liquid for americium recovery from radioactive wastes. Dalton Trans 41 : 6970- 6979.

8. Sengupta A, Mohapatra PK, Iqbal M, Verboom W, Huskens J, et al. (2012) Extraction of Am(III) using novel solvent systems containing a tripodal diglycolamide ligand in room temperature ionic liquids: a 'green' approach for radioactive waste processing. The Royal Society Advances 2: 7492-7500.

9. Mohapatra PK, Sengupta A, Iqbal M, Huskens J, Verboom W, et al. (2013) Highly Efficient Diglycolamide-Based Task-Specific Ionic Liquids: Synthesis, Unusual Extraction Behaviour, Irradiation and Fluorescence Studies. Chemistry A European Journal 19: 3230-3238.
10. Sengupta A, Mohapatra PK, lqbal M, Huskens J, Verboom W, et al. (2013) A diglycolamide-functionalized task specific ionic liquid (TSIL) for actinide extraction: Solvent extraction, thermodynamics and radiolytic stability studies. Separation and Purification Technology 118: 264-270.

11. Ouadi A, Klimchuk O, Gaillard C, Billard I (2007) Green Chem 9: 1160-1163.

12. Ouadi A, Gadenne B, Hesemann J, Moreau JE, Billard I, et al. (2006) TaskSpecific Ionic Liquids Bearing 2-Hydroxybenzylamine Units: Synthesis and Americium-Extraction Studies. Chem Eur J 12: 3074-3081.

13. Odinets IL, Sharova EV, Artyshin OI, Lyssenko KA, Nelyubina YV, et al. (2010) Novel class of functionalized ionic liquids with grafted CMPO-moieties for actinides and rare-earth elements recovery. Dalton Trans 39: 4170-4178.

14. Giridhar P, Venkatesan KA, Srinivasan TG, Rao PRV (2007) Electrochemical behavior of uranium(VI) in 1-butyl-3-methylimidazolium chloride and thermal characterization of uranium oxide deposit. Electrochim Acta 52: 3006-3012.

15. Sengupta A, Murali MS, Mohapatra PK (2013) Role of alkyl substituent in room temperature ionic liquid on the electrochemical behavior of uranium ion and its local environment. J Radio anal Nucl Chem 298: 209-217.

16. Sengupta A, Murali MS, Mohapatra PK (2013) A comparative study of the complexation of Am(III) and Eu(III) with TODGA in room temperature ionic liquid. J Radioanal Nucl Chem 298: 405-412.

17. Rao CJ, Venkatesan KA, Nagarajan K, Srinivasan TG, Rao PRV, et al. (2009) Electrochemical behavior of europium (III) in N-butyl-N-methylpyrrolidinium bis(trifluoromethylsulfonyl)imide Electrochim Acta 54: 4718-4725.

18. Akdogan Y, Heller J, Zimmermann H, Hinderberger D (2010) The solvation of nitroxide radicals in ionic liquids studied by high-field EPR spectroscopy. Phys Chem Chem Phys 12: 7874-7882.

19. Stoesser R, Herrmann W, Zehl A, Strehmel V, Laschewsky A, et al. (2006) ESR Spin Probes in lonic Liquids. Chem Phys Chem 7: 1106-1111.

20. Stoesser R, Herrmann W, Zehl A, Laschewsky A, Strehmel V, et al. (2006 Phys Chem 220: 1309-1342.

21. Strehmel V, Rexhausen $H$, Strauch $P$ (2010) Influence of imidazolium bis(trifl uoromethylsulfonylimide)s on the rotation of spin probes comprising ionic and hydrogen bonding groups. Phys Chem Chem Phys 12: 1933-1940.

22. Mladenova BY, Chumakova NA, Pergushov VI, Kokorin Al (2012) Rotational and Translational Diffusion of Spin Probes in Room-Temperature lonic Liquids. J Phys Chem B 116: 12295-12305.

23. Sengupta A, Mohapatra PK (2012) Supramolecular Chemistry 24: 11771-778.

24. Dietz ML, Dzielawa JA, Laszak I, Young BA (2003) Influence of solvent structural variations on the mechanism of facilitated ion transfer into roomtemperature ionic liquids. Green Chem 5: 682-685.

25. Schreier S, Polnaszek CF, Smith ICP (1978) Biochim Biophys Acta 515: 375-436.

26. Mazzoleni F, Ottaviani MF, Romanelli M, Martini G (1988) J Phys Chem 92 1953-1958. 